\title{
A framework for checking and ranking integrity constraints in a distributed database
}

\begin{abstract}
The essential aim of a database system is to guarantee database consistency, which means that the data contained in a database is both accurate and valid. Checking the consistency of a database state generally involves the execution of integrity tests (query that returns the value true or false) on the database, which verify whether the database is satisfying its constraints or not. The process of checking integrity constraints has proved to be extremely difficult to implement, particularly in distributed database. This paper proposed a framework for checking integrity constraints in a distributed database by utilizing as much as possible the local information stored at the target site. The proposed framework consists of two main processes, namely: (i) simplify the integrity constraints to produce support tests and integrate them with complete and sufficient tests and (ii) select the most suitable test from several alternative tests when an update operation is submitted to the system. Including these processes in the proposed framework has optimized the process of checking the consistency of the distributed database by reducing the amount of data transferred across the network, the amount of data accessed, the number of sites involved, and the number of integrity constraints to be evaluated.
\end{abstract}

Keyword: Distributed database; Integrity constraints; Integrity constraints checking; Integrity tests 